\title{
Reduciendo la corrupción a nivel local
}

\section{M aría González de Asís*}

\section{Introducción}

La corrupción, junto con una efectiva representación democrática, se presentan como unos de los problemas más importantes a los que se enfrentan los gobiernos locales ${ }^{1}$.

El reto para los gobiernos locales es desarrollar sistemas fiables y transparentes que ofrezcan eficientemente servicios públicos. Gracias a su proximidad, el gobierno local tiene mayor oportunidad de cumplir este reto y de controlar la corrupción.

La tendencia actual en los procesos de descentralización es la de dar todo el poder a los gobiernos locales. En general, la descentralización no sólo transfiere recursos a los gobiernos locales, sino que también transfiere autoridad para encontrar esos recursos y para ejecutar su poder discrecional. Tiene el potencial 0 para reducir la corrupción y mejorar el destino de los servicios públicos, o para exacerbar la corrupción y minar dicho destino.

El objetivo de esteartículo es aportar una infraestructura conceptual y ejemplos concretos para controlar la corrupción a nivel local. La infraestructura servirá a los reformadores locales y a los grupos de ciudadanos que tienen interés en cambiar los sistemas corruptos. En él se señalan estrategias para involucrar a la gente en la toma de decisiones y su seguimiento y para identificar las prioridades de la reforma a través de instrumentos para diagnosticar, para desarrollar sistemas y crear procesos que sirvan para mejorar la actuación del gobierno.

En este contexto, la fuerte voluntad política, la voz del pueblo, el apropiado apoyo técnico, y una estrategia realista de implantación a largo plazo, son vitales para el éxito.

Se pueden esperar resultados significativos siempre que se den tres factores: una buena información (con una formulación del plan deacción basado en una profunda evidencia empírica en cada caso), la construcción de coaliciones que desemboquen en una acción colectiva, y un mandato político transparentea nivel local.

\section{Comprensión del problema}

La corrupción distorsiona la distribución de los recursos y la actuación de los gobiernos locales. Las consecuencias de la corrupción son servicios públicos de baja calidad, una aumentada polaridad social, una menor inversión en el municipio y un crecimiento económico disminuido. Luchar contra la corrupción es crucial para los objetivos más amplios de promover el desarrollo económico, la justicia social y unos gobiernos locales más eficaces.

La corrupción recibe distintos nombres alrededor del mundo ${ }^{2}$, aunque no es de ninguna forma exclusiva de una localidad, país o región en particular. La definición general de corrupción empleada en el Banco Mundial es «el abuso de los servicios públicos para obtener una ganancia personal» ${ }^{3}$.

La corrupción implica un comportamiento incorrecto por parte de los cargos públicos, tanto si son políticos como funcionarios, el cual conlleva el abuso del poder que les ha sido confiado, para enriquecerse ellos mismos 0 aquellos próximos a ellos.

La corrupción a nivel local puede aparecer bajo distintas formas. Los tipos principales son: la aceptación, el requerimiento 0 la extorsión de sobornos, el mecenazgo, el nepotismo, el robo de bienes públicos, la corrupción política y el clientelismo. Todos los tipos de corrupción están relacionados con perversos incentivos creados en una institución o en un gobierno local en particular. Los incentivos perversos son una serie de motivaciones que hacen que ciertos individuos actúen contrariamente a las normas de su cargo. En contraste, los incentivos buenos incluyen, entre otros, recompensas, subidas salariales, dignidad y reputación.

La corrupción sistémica dentro del sector público puede ser definida como el uso sistemático del cargo público para el propio beneficio, que reduce la calidad o disponibilidad de los bienes y servicios públicos. En la corrupción sistémica existen vías de dis- 
tribución que mueven los sobornos desde su punto de recaudación hacia arriba a través de la jerarquía institucional. También incluye a la alta corrupción de los funcionarios públicos y de los políticos de alto nivel, quienes toman decisiones que implican a grandes contratos públicos o proyectos. Esta corrupción está motivada por ambición personal, no necesidad, o es usada para beneficiar la recogida de fondos de una partido político. Sus componentes son robo (la privatización espontánea de empresas públicas, el uso incontrolado de los fondos de la empresa, obteniendo créditos sin devolución, pago de salarios a empleados inexistentes, etc., y/o corrupción política), violación de la legislación electoral, financiación extra-legal de campañas, y solución de conflictos parlamentarios a través de modalidades ilegales.

Las causas de la corrupción son muchas y pueden variar de un gobierno local a otro. Sin embargo, algunos factores comunes que contribuyen al abuso del poder público para beneficio propio son: 1) incentivos perversos, 2) falta de información pública y de transparencia; y 3) falta de responsabilidad.

1) La existencia de perversos incentivos institucionales aumenta la probablidad de que exista corrupción. El riesgo de corrupción aumenta a medida que los funcionarios públicos re ciben nuevas responsabilidades y funciones, y también cuántos más funcionarios están expuestos a tener que facilitar servicios de calidad con limitados presupuestos. Los incentivos perversos más comunes son la falta de eficiencia, la discrecionalidad arbitraria, la excesiva complejidad, salarios bajos, la presión de los superiores, impredecibilidad de los procedimientos administrativos usados para proveer servicios públicos (por ejemplo, permisos, pago de impuestos), la baja calidad de la administración pública, la falta de fuentes claves de rentas públicas de la ciudad (tales como los impuestos sobre la propiedad o el «caster fiscal») ${ }^{4}$, los lazos de parentesco existentes, las tradiciones/orgullo civil, y nombramientos/ascensos basados en un sistema no meritorio.

El cobro de las rentas públicas, por ejemplo, disminuye cuando se complica el sistema de evaluación del valor de propiedad. Se han introducido buenos incentivos para solucionar este problema, en municipios tales como La Paz (Bolivia) y Limpio (Paraguay). En estas ciudades, existe una auto-evaluación del sistema, donde los ciudadanos pueden declarar el valor de sus propiedades bajo la amenaza silente de que la ciudad podría adquirir sus propiedades por el doble del valor declarado.

2) La falta de información pública y de transparencia permite a los funcionarios públicos mantener el aura de secretismo necesaria para que la corrupción continúe. Cuando las operaciones gubernamentales son tratadas como secretos municipales, aumentan las oportunidades para la corrupción. El aura de secre tismo que rodea a los gobiernos locales refuerza la percepción pública de las ilegalidades potenciales, aumentando así el incentivo para la corrupción y disminuyendo el incentivo para generar y conservar los recursos municipales.
3) La falta de responsabilidad significa que los funcionarios públicos afrontan poco o ningún «riesgo» de ser castigados por delitos. Sin esta clase de control sobre su comportamiento, son más propensos a verse implicados en la corrupción. Como señaló Benjamin Franklin, «M antener honesto un gobierno y así intactas nuestras libertades requiere eterna vigilancia» ${ }^{5}$. Los funcionarios se ven tentados por la corrupción cuando el beneficio percibido es mayor que el castigo si les atrapan. En este sentido, la corrupción es una opción racional: es un crimen de cálculo ${ }^{6}$.

Conocer la extensión y naturaleza de la corrupción en un municipio ayuda a establecer sus causas y las prioridades de re forma, identificando las actividades y agencias donde se concentra la corrupción. Se utiliza evidencia concreta para despersonalizar el debate sobre la corrupción, para orientar el enfoque hacia asuntos importantes, y para educar al público sobre los costes económicos y sociales de la corrupción. También se establece una línea de referencia contra la que pueden medirse posteriormente los éxitos y fracasos de la reforma. Por eso los estudios diagnósticos son una parte integral para construir el consenso, diagnosticando problemas claves, diseñando programas de acción anticorrupción para los gobiernos locales y evaluando su implantación.

Los programas anti-corrupción a nivel local constituyen un elemento clave dentro de un pás. D ebido a su tamaño y a su proximidad con los ciudadanos, un programa local de anticorrupción permite una mejor adaptación de la experiencia internacional a las condiciones locales, antes de aplicarlo a un nivel nacional. El éxito puede engendrar más éxito, creando un efecto dominó entre los municipios que implanten este tipo de programa.

\section{Estrategias para combatir la corrupción}

El menor tamaño de un municipio es una ventaja para crear una estrategia sistémica y práctica de cooperación entre la sociedad civil y el gobierno, que sea capaz de responder mejor a las necesidades, peticiones y demandas de individuos o grupos de la comunidad.

Las estrategias pragmáticas para combatir la corrupción a nivel local pueden dirigir incentivos perversos a través de un proceso participativo de reforma institucional. Algunas de las mejores experiencias extranjerasse presentan aquí para proporcionar ejemplos concretos de reforma. Cada elemento es crucial; sin embargo, cada uno debería ser implantado en el contexto de una estrategia clara, participativa e integrada, que sea capaz de construir reformas sostenibles a nivel local. 
Construir reformas institucionales sostenibles a nivel local implica dar al gunos pasos hacia el diseño de una estrategia participativa, comenzando con una fuerte voluntad política de reforma. Los programas deberían diseñarse sin enfocarse en la corrupción misma, sino distribuyendo los recursos municipales de una manera más justa para la reforma. Para dar prioridad a áreas para la reforma, el gobierno local y la sociedad civil, deberían utilizarse grupos de trabajo participativos cuyos cometidos sean identificar los problemas y los incentivos perversos y después proponer formas para monitorizar y evaluar las reformas.

\section{Voluntad política}

Una fuerte voluntad política en los niveles más altos del gobierno local es una precondición para iniciar una reforma anticorrupción. La voluntad política normalmentesurge con un cambio en el gobierno (por ejemplo, gobiernos recién formados 0 elecciones), el cual proporciona una oportunidad para la reforma. Esta oportunidad permite a los reformadores de dentro y fuera del gobierno diseñar una estrategia conjunta de reforma.

\section{Instrumentos para el diagnóstico y grupos de trabajo}

Los estudiosson una entrada deinformación clavepara diseñar una estrategia de reforma y también ayudan a aumentar la conciencia sobre el asunto. Los estudios de las familias, de las empresas y delosfuncionarios públicos, identifican los principalesproblemas gubernamentales en el municipio y ayudan a establecer prioridades para la refoma. Sin embargo, es crucial encontrar una empresaindependientepara ejecutar los estudios y evitar la posibleinterferencia política durante la recolección de los datos. Por ejemplo, un estudio sobre la percepción del cliente recoge información sobre las percepciones de la empresa y de los ciudadanos sobre el servicio de reparto, así como dela integridad y credibilidad del gobierno municipal. El estudio mide las percepciones de eficiencia, eficacia, corrupción, y de la calidad y amplitud de la información provista por las autoridades locales para uso público. Los estudios pueden prestar credibilidad al esfuerzo reformador y facilitar el impulso necesario para conseguir un progreso significativo del control de la corrupción y de la reforma del gobierno.

\section{D iagnóstico participativo: grupos de trabajo y plan de acción}

Los resultados del estudio se presentan a un grupo de trabajo al que asisten tanto los funcionarios locales como los miembros de la sociedad civil. Basándose en los hallazgos del estudio, los grupos de trabajo establecen prioridades de reforma y después proponen formas innovadoras para dirigirlas. En el plan de acción exponen los actores y recursos que se necesitan para implantar las reformas municipales. Consecuentemente, los grupos de trabajo pueden monitorizar y evaluar periódicamente la implantación de las reformas.

\section{Instrumentos para luchar contra la corrupción}

Aunque no existe ninguna receta para luchar contra la corrupción a nivel local, los instrumentos presentados más abajo pueden usarse selectivamente para diseñar una estrategia de lucha contra la corrupción.

\section{Simplificación de los procedimientos administrativos}

La complejidad e impredecibilidad de los procedimientos administrativos crean confusión y dan a losfuncionariospúblicosuna discrecionalidad arbitraria para desarrollar sus obligaciones. LoS manuales administrativos para disminuir y estandarizar los procedimientos y el sistema informatizado para almacenar la información administrativa, incluyendo los derechos de registro y las estimaciones del costedelos procedimientos administrativos, pueden combatir este problema. D ichos instrumentos pueden mejorar la coordinación entre departamentos, evitar la duplicación de obligaciones, eliminar las decisiones arbitrarias y acelerar el trabajo administrativo. A través de Internet, por ejemplo, los ciudadanos pueden tener acceso a una serie de pasos que son necesarios para obtener documentos. D espués de que varios municipios reformaran sus procedimientos administrativos, aumentó la satisfacción de losciudadanos con los serviciosy las quejas disminuyeron en al gunos casos hasta más del setenta por cien (por ejemplo, en Campo Elías, Venezuela).

Sin embargo, para tener el máximo efecto, los ciudadanos de ben estar bien informados acerca de dichos procedimientos y de cómo beneficiarse de ellos. Una oficina de información puede dar a los ciudadanos esa información y asistencia. Una vez quelosciudadanos conocen las tarifas que pueden exigir los proveedoresy los pasos que deben seguir siempreque están implicados los procedimientosadministrativos, pueden exigir mejor los serviciosalosque tienen derecho. En municipios tales como La Paz (Bolivia) o San $M$ arcos (Guatemala), todas las tarifas municipales de los servicios están expuestas en las paredes delas áreas públicas para impedir que los empleados municipales cobren de más por sus servicios.

\section{Participación ciudadana}

Ciertas medidas para aumentar la participación ciudadana en el gobierno pueden promover la transparencia y la responsabili- 
dad en la administración local. Estas medidas pueden institucionalizarse a través de estatutos locales, dando a los ciudadanos tanto el incentivo como los medios jurídicos para participar en el gobierno local.

a) Asambleas públicas. Una de las formas más útiles de aumentar la participación ciudadana es a través de asambleas públicas. En muchos municipios, esto ha proporcionado un foro efectivo para desarrollar el presupuesto anual. Las asambleas públicas en la elaboración presupuestaria ayudan a identificar y a dar prioridad a las necesidades de los ciudadanos; a la co-gestión de los recursos municipales; al control y la evaluación de los compromisos del alcalde; y crean oportunidades para la participación, la responsabilidad y la transparencia. A través de la puesta en práctica del presupuesto participativo, la sociedad civil puede convertirse en una parte integral para definir las prioridades y determinar los compromisos. Así, los ciudadanos se sienten más satisfechos con los servicios municipales y están capacitados para preocuparse sobre proyectos y para monitorizar su actuación. Además, los ciudadanos pueden ver que reduciendo la corrupción, se liberan los recursos financieros que de esta forma pueden ser asignados a servicios esenciales.

\section{PRESU PUESTO PARTICIPATIVO DE PORTO ALEGRE}

Uno de los mejores ejemplos de participación ciudadana del presupuesto es el caso de Porto Alegre, Brasil. El sistema de presupuesto participativo creado en Porto Alegre es un instrumento que combina la democracia directa con la capacidad y promoción de la representación parlamentaria. En grandes asambleas, los ciudadanos deciden las prioridades del gasto. Las asambleas también eligen dos representantes por cada una de las 16 áreas en que se encuentra dividida la ciudad, que discutirán poste riormente los ingresos y gastos de la ciudad y el criterio de distribución de los recursos. Los criterios (que pueden ser modificados) establecidos para la distribución de los recursos son: carencia de infraestructuras en la zona; población en zonas con grandes carencias de infraestructuras viarias, número de población y zonas marginadas.

En Porto Alegre, los ciudadanos identifican y priorizan sus necesida des, cogestionan los recursos municipales y evaluan los objetivos propuestos; todo ello hace que los ciudadanos sean más responsables y exista en la gestión municipal mayor responsabilidad y transparencia.

Aunque existen distintas formas de conducir a las asambleas públicas sobre presupuestos, normalmente el alcalde presenta un presupuesto preliminar a los ciudadanos. Utilizando las cifras del presupuesto preliminar, los ciudadanos se dividen en grupos de trabajo para debatir y priorizar sus necesidades. Entonces, se ce lebran los talleres participativos en materia presupuestaria en cada barrio, para así determinar sus respectivas listas de prioridades. Estas listas se presentan a los técnicos en un taller participativo, se discuten los proyectos individuales y se comprueba si son factibles y su costo. Las asociaciones vecinales presentan propuestas revisadas al municipio, y una comisión tripartita compuesta por ciudadanos, representantes del ayuntamiento y funcionarios pú- blicos, seleccionan los proyectos. Cada tres meses, el alcalde debe presentar un escrito público dando cuenta del estado del presupuesto y de los proyectos incluidos en él.

Los requisitos básicos para una asamblea pública de temas presupuestarios son: 1) un gobierno democrático y transparente, dirigido por un alcalde que esté dispuesto a gobernar de forma transparente; 2) el desarrollo y apoyo de asociaciones vecinales; 3) la inversión en la educación cívica; y 4) la voluntad polítca y la capacidad técnica de conducir las asambleas públicas sobre temas presupuestarios.

b) Informatización de los datos y sistemas electrónicos. 0 tro mecanismo para mejorar la responsabilidad y transparencia es permitiendo el acceso a la información a través de sistemas de datos informatizados. Se puede diseñar e implantar un conjunto de software para que los ciudadanos puedan acceder a la información y a los procedimientos llevados a cabo por el municipio. Los individuos pueden visitar una oficina de Participación Ciudadana y, si es necesario, recibir apoyo del personal para poder conocer los procedimientos municipales por medio del ordenador, incluyendo las subastas públicas, las adqusiciones y la ejecución de las obras públicas. Este instrumento pretende conseguir un gobierno más transparente a través del control público y de la informatización de los procedimientos.

Cada vez más municipios de todo el mundo están implantando sistemas de datos informatizados a través de las páginas de Internet. Los servicios varian de un municipio a otro. Por ejemplo, los gobiernos locales de Porto Alegre (Brasil), Limpio (Paraguay), M orón (Argentina) y O bnisnsk (Rusia) facilitan servicios públicos a través de Internet. Esto no sólo permite a los funcionarios locales un acceso fácil a la información, sino que también permite a los ciudadanos usar las terminales de ordenadores en el ayuntamiento 0 en las oficinas de información pública para comprobar esa información, denunciar casos de corrupción, o pedir determinados permisos o licencias. 0 tro ejemplo es el colocar el presupuesto del año anterior en su página de Internet y el proyecto de presupuesto para el año siguiente, incluyendo los impuestos, las licencias y otros servicios. Estos servicios fomentan la participación en el gobierno local.

O tro ejemplo son los servicios electrónicos, que permiten que los negocios compitan en un campo de juego más nivelado, dado que pueden comparar precio y niveles de calidad en Internet. Por ejemplo, Campo Elías (Venezuela) ha implantado un servicio de Corrupción Electrónica (E-C orruption). Es un servicio tecnológico que permitea los ciudadanos visual izar las transacciones municipales a través de Internet. Toda empresa que proporcione bie nes 0 servicios de parte del gobierno municipal (por encima de un umbral de coste) tiene que informar en la página de Internet. Si no lo hiciera, sería sancionada. Aquellos ciudadanos que no dispongan de acceso a Internet, podrán utilizarlo en la 0 ficina de Información. 
c) O ficina para la Participación Ciudadana. U na oficina para la colaboración ciudadana es un complemento de los sistemas de datos, las asambleas y los talleres, para promover la participación ciudadana. Esa oficina: 1) crea canales para el diálogo entre el gobierno y la sociedad civil; 2) proporciona un lugar real para que los ciudadanos se involucren en las actividades participativas; 3) organiza y facilita información sobre los servicios municipales, sobre los funcionarios y los procedimientos administratiVos; 4) responde a las quejas de los ciudadanos de una forma conveniente; y 5) sirve como punto focalizado para organizar las campañas anti-corrupción.

d) Comisiones de control de gasto. 0 tra forma utilizada de acercarse a los ciudadanos para alcanzar la responsabilidad y la transparencia es la creación de comisiones de control de gasto, compuestas por ciudadanos, concejales, y funcionarios locales. Una comisión típica incluiría un ciudadano representando a cada asociación vecinal, un concejal, y dos funcionarios municipales elegidos por el alcalde. El objetivo de la comisión es facilitar la participación de la comunidad; mantenerla informada sobre el proceso del presupuesto; participar en talleres técnicos y elegir los proyectos de obras públicas; desarrollar un calendario y un modo (ya sea por administración directa o por contrato) para la ejecución de las obras públicas y vigilar las obras públicas y los programas comunitarios. Los miembros de la comisión son nombrados normalmente por un año, y tienen cuatro reuniones públicas con los ciudadanos al año. Un ejemplo de comisión de cuentas local se encuentra en Campo Elías (Venezuela).

También encontramos otros ejemplos de comisiones de control de gasto a nivel local en otras ciudades como la Provincia de M endoza (Argentina) o en Limpio (Paraguay). En M endoza, el gobierno organizó una asamblea pública, con la ayuda de la Fundación del Poder Ciudadano. El objetivo de la asamblea pública era revisar los documentos de licitación de las compañías para asegurarse de que las condiciones de contratación eran transparentes y limitar la arbitrariedad de los funcionarios públicos. El gobierno recibió las propuestas de una forma transparente, y después las evaluó con un equipo imparcial de expertos. Era importante contar con políticos, líderes sindicales, ciudadanos, analistas privados y otros expertos, además de periodistas, participando u observando la asamblea.

0 tro exitoso ejemplo es el implantado en Morón (Argentina). «El Programa para la Contratación Transparente» (PCT) del Poder Ciudadano en Moron ha combinado dos componentes: celebrar asambleas públicas donde la autoridad responsable se reúne con ciudadanos, empresas, expertos, y representantes de la oposición, para manifestar sus objeciones y sus sugerencias sobre los términos de la contratación; y la firma de un Pacto de Integridad donde tanto el gobierno como todas las empresas que compiten por el proyecto comparten un control recíproco para impedir el pago de sobornos entre los ofertantes y el Estado.

\section{Control y evaluación}

M ás allá de las técnicas internas de control, tales como las asambleas públicas sobre materia presupuestaria, los sistemas de control informatizado de las obras públicas, y los talleres locales o las audiencias públicas, los instrumentos externos de control son tremendamente importantes en el proceso de reformas institucionales.

Los seminarios públicos proporcionan una forma efectiva de controlar, evaluar y seguir el proceso de implantación de los programas municipales. Por el hecho de asistir a los seminarios, los ciudadanos son capaces de proporcionar una retroalimentación al gobierno local sobre el esfuerzo de la reforma. Específicamente, retroalimentan a la oficina de participación ciudadana, a la comisión de control del gasto, al sistema informatizado de datos y a las ordenanzas municipales. Los funcionarios de otras localidades también pueden beneficiarse de los seminarios, aprendiendo cómo aplicar las reformas en sus respectivos muncipios.

Siguiendo la fase de implantación, el estudio de la percepción de un segundo cliente debería ser utilizado para evaluar el impacto del programa municipal de reforma. Los resultados de este estudio deberían mostrar que las metodologías basadas en el consenso usadas para implantar la reforma han aumentado la transparencia, el acceso público a los servicios y la capacidad gubernamental para solucionar los problemas municipales. Comparando los resultados de los dos estudios, deberíamos ver mejoras en los indicadores que miden la eficiencia, el acceso y la transparencia.

\section{Conclusión}

Si redunda en éxito, los esfuerzos para erradicar la corrupción potencian la actuación y legitimidad del sector público. Al perseguir la transparencia y la responsabilidad, la reforma permite a los ciudadanos y a los gobiernos locales gobernar, utilizar los recursos, proporcionar los servicios y aumentar la mayor parte de la calidad de vida de la comunidad, más efectivamente. Las reformas que tienen efectos más a largo plazo incluyen procedimientos administrativos simplificados, un aumento de la participación ciudadana en el gobierno y sistemas de monitorización y de evaluación.

Estos esfuerzos dependen de distintos elementos. En primer lugar, el compromiso político es indispensable. El compromiso político a nivel local es una condición crucial para al canzar reformas institucionales comprensibles y, en particular, para crear sistemas responsables y transparentes y para erradicar la corrup- 
ción. En segundo lugar, la información es clave para establecer prioridades y para dar credibilidad al esfuerzo reformador. LoS estudios proporcionan datos solidos para diseñar, monitorizar y evaluar las reformas. En tercer lugar, la implicación de la sociedad civil en las reformas es vital para su legitimidad y su viabilidad. El consenso alcanzado en los talleres refuerza el compromiso para el plan de acción y la posibilidad de una implantación con exito.

La información es un instrumento esencial para lograr responsabilidad, transparencia y participación. La reforma de los sistemas de información ha tenido un impacto significativo en el gobierno y en la sociedad civil. Una condición vital para cons- truir sistemas eficientes, responsables y transparentes a nivel local, es la simplificaión y accesibilidad de la información. Los talleres participativos, las campañas de información y el sistema informatizado de datos de la 0 ficina de Información, proporcionan un flujo corriente de información accesible para el consumo público, aumentando aśl la probabilidad de que exista transparencia y responsabilidad.

La experiencia en muchos municipios del mundo ha demostrado el poderoso efecto del compromiso político, de la capacidad técnica para diseñar e implantar reformas y de una fuerte unión con la sociedad civil, en el effuerzo por potenciar la eficiencia, la equidad y la transparencia en el gobierno local.

\section{Notas}

Artículo traducido por Laura AlBa M uñoz.

* Instituto del Banco M undial.

${ }^{1}$ Decentralization and Local Government Performance. Jean-Paul Faguet. Centre for Economic Performance and D evelopment Studies Institute. L ondon School of E conomics, 1998. Los ciudadanos a menudo creen que el soborno es la forma más efectiva de pedir y recibir servicios, y ven el sector público como una institución para el enriquecimiento personal. M ás aún, los ciudadanos no están motivados para participar en el sector público.

${ }^{2}$ Terminos locales para la corrupción son: dash en África occidental, la bustarella en Italia, grease en América, graft en Nueva Zelanda, y mordida en M éico.

${ }^{3}$ TheWorld Bank, Helping Countries Combat Corruption: The Role of theWorld Bank, Poverty Reduction and Economic M anagement Network, September 1998. Ver páginas 19-20 para la definición de corrupción.
${ }^{4}$ Los impuestos son cruciales para la gestión, planificación y regulación urbana.

5 Citado de la Convención Constitucional de Philadelphia de 1787.

6 Robert KlitgaARd, Rondald M ACLEan-Abaroa y H. LIN DSEY PARRIS, «Corrupt Cities», Bentham (1843, p.399 [1788]) «... theprofit of thecrimeistheforcewhich urges man to delinquency: the pain of the punishment istheforceemployed to restrain him from it. If the first of these for ces be the greater, the crime will be committed; if the second, the crime will not be committed». Crimeand Punishment by BECKER (1968). Sugiere que ciertos criminales considerarán los beneficiosy costes derivados desu compromiso, así como los beneficios y costes derivados de abstenerse de su compromiso. BECKER, G ary S. (1968) «C rime and Punishment: An Economic Approach», Journal of Political Economy 76: 169-217; BECCARIA, C. (1971) «0 n crime and punishment (1764)», en S.E. G RU pp cd., Theories of Punishment, Bloomington (IN ), Indiana University Press, 117-137; Benth am, Jeremy (1843) «Principles of Penal Law», Works, 1:399 ff.

\section{Bibliografía}

Enhancing Integrity and Accountability. Preventive-corruption M easures for Municipalities in Central America. N oviembre, 1998.

U rban M anagement ProgrammeWorking Paper no. 7. «A Practical Approach to D ealing With M unicipal M alfeasance» by R. K litgaard, R. M acLean-Abarao and H. Lindsey
Parris (M ay 1996), jointly published by UNDP, UNCH S (H abitat) and the World Bank.

Corrupt Cities. R. Klitgaard, R. Maclean-Abaroa and H. Linsdey Parris. ICS Press and the World Bank Institute. 2000. Reducing Corruption: Lessons from Venezuela. 